\title{
The impact of transcutaneous electrical nerve stimulation on COVID-19 patients, especially those admitted to the intensive care unit
}

Sir,

The rapid spread of COVID-19 has made it important to have different and effective therapeutic strategies. Medical system has three major components which contains prevention, treatment, and rehabilitation. There are no clinically approved specific therapeutic drugs or vaccines available for COVID-19 patients up to present time. Most of the treatments are symptomatic and supportive including antiviral and anti-inflammatory drugs. Antiviral drugs and vaccines are currently under evaluation and development. Due to potential rapid worsening of hypoxemia, COVID-19 patients may need to be admitted in intensive care unit (ICU). Therefore, nonpharmaceutical interventions and rehabilitations such as physiotherapy, early mobilization and exercise are vitally necessary to please treat severe COVID-19 patients. Physiotherapy is effective in strengthening of respiratory muscles, reduction of inflammation, enhancing immune function and also helps in mucus easily removal and relieving various symptoms of COVID-19 patients. $^{1}$

Transcutaneous electrical nerve stimulation (TENS) is a kind of physiotherapy and a non-pharmaceutical intervention that uses electric current to activate nerves for therapeutic reasons. Electrical currents pass through the intact surface of the skin to activate underlying nerves. TENS has few complications and side effects compared to many medications and has no potential for overdose. It is useful for maintenance and improvement of muscle function especially in ICU admitted patients in which they cannot perform voluntary resistance training.

Due to following reasons, we recommend investigating TENS effects on COVID-19 patients:

Respiratory symptoms and dyspnea are the main manifestation and a serious problem for COVID-19 patients. Many studies reported that TENS alleviate dyspnea and lung function and improve $\mathrm{FEV}_{1} \%$ predicted in patients with chronic obstructive pulmonary disease (COPD) and asthma that may cause by elevation in betaendorphin levels. ${ }^{2,3}$

COVID-19 patients may suffer from pain in peripheral and central muscles such as chest wall pain, which may reduce the healing process. Several studies have shown that TENS is used to treatment and reduction of acute and chronic pain conditions. It can also reduce post-operative chest pain due to median sternotomy after cardiac surgery.

The entrance of severe acute respiratory syndrome coronavirus 2 (SARS-CoV-2) into alveolar epithelial cells, results in cytokine storm syndromes that can causes acute respiratory distress syndrome (ARDS) and multiple organ failure. Therefore, treatment of the cytokine storm syndrome is an important part of rescuing severe COVID19 patients. Interleukin-6 plays an important role in inducing cytokine storm syndrome. Some studies showed that serum interleukin-6 levels decrease because of rehabilitation with TENS. ${ }^{4}$

Patients with COVID-19 may have neurological disorders such as muscle spasticity and neuralgia. TENS is also recommended as a treatment for muscle spasticity.

Advanced progressive disease may cause for prolonged immobilization, which results in neuromuscular complications and muscle weakness. Patients with COVID-19 may need to be admitted in ICU due to critical care triage need. Muscle weakness because of SARS-CoV2 infection and ICU acquired weakness (ICU-AW) due to intensive medical management for some COVID-19 patients such as prolonged protective lung ventilation, sedation and use of neuromuscular blocking agents may worsen their morbidity and mortality. Similar to it, low serum iron levels were detected in COVID-19 patients which is related with skeletal muscles weakness. ${ }^{5}$ TENS improves critical illness polyneuropathy/myopathy, prevents reduction in thickness of chest muscle, preserve protein synthesis in muscle and decrease admission period in ICU-admitted patients. ${ }^{6,7}$ Also, several studies have reported that electrostimulation treatment improves muscle strength, the exercise capacity, functionality and fatigue and also is able to decelerate ambulatory muscle weakness in COPD patients. ${ }^{8,9}$

\section{CONCLUSION}

As mention above, patients with COVID-19 may suffer from many symptoms and complications that some of them are caused by prolonged immobilization especially in ICUadmitted patients. Based on our review on electrical stimulation, TENS may be a potential and effective therapeutic method with few side effects compared to many medications to relieve various symptoms and complications of COVID-19 patients. 


\section{Rasoul Alipour ${ }^{1,2,3 *}$, Fariba Mikaeili ${ }^{4}$}

${ }^{1}$ Emergency Department, Alinasab Hospital,

${ }^{2}$ Treatment Management of East Azerbaijan,

${ }^{3}$ Social Security Organization, Tabriz, Iran

${ }^{4}$ Midwifery Unit, Islamic Azad University of Ardabil, Iran

*Correspondence to

Dr. Rasoul Alipour,

E-mail: rasoul.alipour.med@gmail.com

\section{REFERENCES}

1. Feng F, Tuchman S, Denninger JW, Fricchione GL, Yeung A. Qigong for the Prevention, Treatment, and Rehabilitation of COVID-19 Infection in Older Adults. Am J Geriatr Psychiatry. 2020;28(8):812-9.

2. Ngai SP, Jones AY, Hui-Chan CW, Ko FW, Hui DS. Effect of Acu-TENS on post-exercise expiratory lung volume in subjects with asthma-A randomized controlled trial. Respir Physiol Neurobiol. 2009;167(3):348-53.

3. Ngai SP, Jones AY, Hui-Chan CW, Ko FW, Hui DS. Effect of 4 weeks of Acu-TENS on functional capacity and beta-endorphin level in subjects with chronic obstructive pulmonary disease: a randomized controlled trial. Respir Physiol Neurobiol. 2010;173(1):29-36.

4. Akar O, Günay E, Ulasli SS, Ulasli AM, Kacar E, Sariaydin M, et al. Efficacy of neuromuscular electrical stimulation in patients with COPD followed in intensive care unit. Clin Respir J. 2017;11(6):74350 .
5. Alipour R, Hashemi SH, Mikaeili F. Serum iron level in patients with COVID-19: a case report study. Int J Res Med Sci. 2020;8:2658-62.

6. Connolly B, O'Neill B, Salisbury L, Blackwood B. Enhanced Recovery After Critical Illness Programme Group. Physical rehabilitation interventions for adult patients during critical illness: an overview of systematic reviews. Thorax. 2016;71(10):881-90.

7. Dall' Acqua AM, Sachetti A, Santos LJ, Lemos FA, Bianchi T, Naue WS, et al. Use of neuromuscular electrical stimulation to preserve the thickness of abdominal and chest muscles of critically ill patients: A randomized clinical trial. J Rehabil Med. 2017;49(1):40-8.

8. Lopez Lopez L, Granados Santiago M, Donaire Galindo M, Torres Sanchez I, Ortiz Rubio A, Valenza MC. Efficacy of combined electrostimulation in patients with acute exacerbation of COPD: randomised clinical trial. Med Clin (Barc). 2018;151(8):323-8.

9. Kaymaz D, Ergün $\mathrm{P}$, Demirci E, Demir N. Comparison of the effects of neuromuscular electrical stimulation and endurance training in patients with severe chronic obstructive pulmonary disease. Tuberk Toraks. 2015;63(1):1-7.

Cite this article as: Alipour R, Mikaeili F. The impact of transcutaneous electrical nerve stimulation on COVID-19 patients, especially those admitted to the intensive care unit. Int J Adv Med 2020;7:1612-3. 